\title{
VLE calculations by applying a modified perturbed hard sphere EOS
}

\author{
Min-Lon Yu, Yan-Ping Chen * \\ Department of Chemical Engineering, National Taiwan University, Taipei, Taiwan.
}

Received 22 November 1995; accepted 22 August 1996

\begin{abstract}
An equation of state (EOS), developed by using an improved expression of the coordination number model of square-well fluids, was applied in vapor-liquid equilibrium (VLE) calculations of real fluids and their mixtures. The EOS was written in a perturbed hard sphere form which had three parameters for each pure fluid. In this work, the EOS parameters for pure nonpolar and polar fluids were determined. It is demonstrated that the pure fluid properties were satisfactorily correlated and the EOS parameters showed reasonable physical significance. In the VLE calculations of fluid mixtures, the results were again satisfactory and were superior to those from commonly used cubic-type EOS. It is indicated that this modified perturbed hard sphere (MPHS) EOS yielded significantly better VLE calculation results on asymmetric systems of nonpolar + polar binary mixtures. The scattering of the binary interaction parameters of this MPHS-EOS was less than that from other EOSs. Ternary calculations by directly applying the binary parameters were also successful. (C) 1997 Elsevier Science B.V.
\end{abstract}

Keywords: Theory; Equation of state; Vapor-liquid equilibria; Mixture

\section{Introduction}

The square-well (SW) potential function has been widely used in developing equations of state (EOS) from statistical thermodynamics. This potential function is simple and reasonable to model the real fluid behavior and many computer simulation data are available in applying this potential function. Thermodynamic properties of SW fluids are dependent on the molecular interactions, the well depth, and the microscopic structure which is usually expressed by the coordination number model. By incorporating the coordination number model and the generalized van der Waals partition function (Sandler, 1985), a theoretically-based EOS can then be developed. Lee et al. (1985, Lee et al., 1986, Lee and Sandler, 1987, Lee et al., 1989) have proposed a coordination number model and

\footnotetext{
* Corresponding author.
} 
the corresponding EOS in their respective works. Lee and Chao (1987) and Guo et al. (1990a,Guo et al., 1990b) have also presented their coordination number models and Guo et al. (1990b) have derived their EOS. Some of these EOS cannot be employed in fluid mixture calculations owing to the complexity of the coordination number models and this has limited their applications in engineering computations.

A new coordination number model for SW fluids was proposed in our previous study (Yu and Chen, 1995). This new coordination number model, which included a density-dependent correction term to the low density expression, satisfied the low density and the close-packed limiting requirements, and agreed well with the molecular simulation results at intermediate densities. This new model has been tested with the molecular simulation data on both pure and mixture SW fluids, and better results have been obtained in comparison with those from other coordination number models. A new EOS, written in a modified perturbed hard sphere (MPHS) form, was developed from our coordination number model. In our previous work, the MPHS-EOS showed good results for the compressibility factor calculations of SW fluids and their mixtures. This EOS was also used for real $n$-paraffins as a preliminary study in our previous work and satisfactory correlations on vapor pressures and liquid densities were observed. In this study, we extended our calculations to other nonpolar and polar pure fluids and applied the MPHS-EOS for vapor-liquid equilibrium (VLE) calculations on binary and ternary mixtures. We compared the VLE calculation results from the MPHS-EOS with those from the commonly used cubic-type EOS of Peng and Robinson (1976) and Patel and Teja (1982) to test the applicability of the MPHS-EOS in phase equilibrium computations.

\section{The MPHS equation of state and pure component parameters}

A new coordination number model has been proposed in our previous work (Yu and Chen, 1995) where the coordination number at any density is written as a product of the low density limit expression and a correction term. The correction term was correlated in our previous work using molecular simulation data of SW fluids. For a mixture of unlike fluids $i$ and $j$, the coordination number $N_{i j}$ is written as

$$
N_{i j}=\frac{4 \pi}{3}\left(\lambda^{3}-1\right) x_{i} \rho \sigma_{i j}^{3} \exp \left(\frac{\epsilon_{i j}}{k T}\right)\left\{1+\left[1.45 \exp \left(\frac{-\epsilon_{i j}}{k T}\right)-1\right]\left[1-\exp \left(1-\phi_{i j}\right)\right]\right\}
$$

where

$$
\phi_{i j}=\frac{\sqrt{2}+\rho \sigma_{i j}^{3}}{\sqrt{2}-\rho \sigma_{i j}^{3}}
$$

and the cross terms of the SW potential function parameters are

$$
\begin{aligned}
& \sigma_{i j}=\frac{1}{2}\left(\sigma_{i i}+\sigma_{j j}\right) \\
& \epsilon_{i j}=\left(\epsilon_{i i} \epsilon_{j j}\right)^{0.5}
\end{aligned}
$$


The coordination numbers of the SW fluids calculated from this model have been compared with those from the molecular simulation data and satisfactory agreements have been observed ( $\mathrm{Yu}$ and Chen, 1995). Applying this coordination number model, an EOS was then derived using the generalized van der Waals theory and the canonical partition function (Sandler, 1985).

Using the Carnahan-Starling EOS (Carnahan and Starling, 1969) for the repulsive term, a modified perturbed hard sphere (MPHS) EOS was developed for a pure fluid (Yu and Chen, 1995)

$$
\begin{aligned}
Z= & \frac{1+\zeta+\zeta^{2}-\zeta^{3}}{(1-\zeta)^{3}}-\frac{4.75}{3} \pi \rho \sigma^{3}\left[\frac{1.45 \epsilon}{k T}-\exp (1-\phi)\left[\frac{1.45 \epsilon}{k T}+1-\exp \left(\frac{\epsilon}{k T}\right)\right]\right. \\
& \left.\times\left[\frac{2 \sqrt{2}-\rho \sigma^{3}-\sqrt{2} \phi}{\sqrt{2}-\rho \sigma^{3}}\right]\right]
\end{aligned}
$$

where $\zeta\left(=\pi \rho \sigma^{3} / 6\right)$ and $\phi$ are two dimensionless functions of the reduced density. In the MPHS-EOS, $\rho$ is the number density, and $\sigma$ and $\epsilon / k$ are the molecular diameter and the interaction energy between molecules, respectively. A temperature-dependent function was introduced into the energy parameter for real fluid calculations and it is expressed as

$$
\frac{\epsilon}{k}=\left(\frac{\epsilon}{k}\right)_{0}\left[1+m\left(1-T_{\mathbf{r}}^{0.5}\right)\right]^{2}
$$

where $(\epsilon / k)_{0}$ is the value of $(\epsilon / k)$ at the critical temperature and $T_{\mathrm{r}}$ is the reduced temperature. Parameter $m$ characterizesd the temperature dependence of $(\epsilon / k)$. For each pure fluid, there are three parameters $\sigma,(\epsilon / k)_{0}$ and $m$ in the MPHS-EOS. These parameters were evaluated by fitting the saturated properties of pure fluids. The following objective function was minimized in this work to obtain the optimal pure fluid parameters

$$
Q=\Sigma\left[\left(\frac{P^{\mathrm{vp}, \mathrm{cal}}-P^{\mathrm{vp}, \exp }}{P^{\mathrm{vp}, \exp }}\right)^{2}+\left(\frac{V^{\mathrm{L}, \mathrm{cal}}-V^{\mathrm{L}, \exp }}{V^{\mathrm{L}, \exp }}\right)^{2}\right]
$$

where the summation is over all data points on the saturation curve. Table 1 lists the MPHS-EOS parameters for typical pure fluids. Table 1 also shows the deviations in vapor pressure and saturated liquid molar volume calculated by the MPHS-EOS on these typical pure fluids. Generally, this EOS gave satisfactory results. More data have been tested in this study where the grand absolute average deviations of $0.66 \%$ in vapor pressure and $1.60 \%$ in saturated liquid molar volume over 2199 data points were obtained. For nonpolar and weakly polar components, it was observed that the pure component parameters in the MPHS-EOS showed regular correlations with their characteristic properties. Fig. 1 presents a plot of $(\epsilon / k)_{0}$ against the critical temperatures for nonpolar and weakly polar pure fluids which exhibited a nearly linear relationship with a constant slope of 0.832 . This value is in agreement with the theoretical values determined from other coordination number models or molecular dynamics simulation data (as shown by Guo et al., 1990b) showing that the slope is between 0.775 and 0.84 for a pure SW fluid. Fig. 2 shows a plot of the effective hard core volumes (equal to $\pi \sigma^{3} / 6$ ) against the van der Waals volumes for nonpolar and weakly polar pure fluids, and this plot also has a linear relationship. The van der Waals volumes were determined from a group 
Table 1

Pure component parameters of the MPHS-EOS

\begin{tabular}{|c|c|c|c|c|c|c|c|c|}
\hline \multirow[t]{2}{*}{ Component } & \multirow{2}{*}{$\begin{array}{l}\text { No. of } \\
\text { data pts. }\end{array}$} & \multirow{2}{*}{$\begin{array}{l}\text { Temp. } \\
\text { range/K }\end{array}$} & \multirow{2}{*}{$(\epsilon / \kappa)_{0}$} & \multirow[t]{2}{*}{$\sigma$} & \multirow[t]{2}{*}{$m$} & \multicolumn{2}{|c|}{ AAD\% } & \multirow[t]{2}{*}{ Data sources } \\
\hline & & & & & & $\overline{V^{\mathrm{L}}}$ & $P$ & \\
\hline Methane & 44 & $91 \sim 187$ & 152.68 & 3.49 & -0.041 & 0.94 & 0.97 & 1 \\
\hline Butane & 27 & $223 \sim 392$ & 344.61 & 4.77 & 0.140 & 0.67 & 0.20 & 1 \\
\hline Octane & 30 & $320 \sim 552$ & 466.86 & 5.92 & 0.310 & 1.27 & 0.43 & 1 \\
\hline Hexadecane & 41 & $422 \sim 594$ & 608.88 & 7.49 & 0.548 & 1.77 & 0.19 & 2 \\
\hline 2-Methylpentane & 23 & $289 \sim 480$ & 404.24 & 5.39 & 0.214 & 0.52 & 0.51 & 1 \\
\hline Cyclopropane & 34 & $197 \sim 386$ & 322.12 & 4.11 & 0.074 & 0.99 & 0.35 & 1 \\
\hline Ethene & 36 & $136 \sim 277$ & 226.75 & 3.82 & 0.042 & 0.93 & 0.57 & 1 \\
\hline 1-Hexene & 33 & $283 \sim 334$ & 412.91 & 5.29 & 0.203 & 0.82 & 0.15 & 1 \\
\hline Ethanol & 25 & $279 \sim 471$ & 431.18 & 4.14 & 0.470 & 2.71 & 2.90 & 1 \\
\hline 1-Propanol & 23 & $305 \sim 492$ & 436.91 & 4.53 & 0.542 & 1.46 & 0.81 & 1 \\
\hline 1-Butanol & 25 & $320 \sim 523$ & 453.12 & 4.87 & 0.556 & 1.39 & 1.56 & 1 \\
\hline Benzene & 42 & $284 \sim 550$ & 455.39 & 4.78 & 0.158 & 1.23 & 0.36 & 1 \\
\hline Toluene & 26 & $301 \sim 537$ & 484.68 & 5.11 & 0.184 & 1.64 & 0.49 & 1 \\
\hline Carbon dioxide & 37 & $217 \sim 297$ & 244.94 & 3.42 & 0.186 & 0.83 & 0.29 & 3 \\
\hline Fluorine & 18 & $95 \sim 140$ & 114.61 & 3.05 & 0.033 & 0.90 & 0.49 & 3 \\
\hline Water & 17 & $313 \sim 473$ & 567.70 & 2.80 & 0.134 & 3.28 & 1.82 & 3 \\
\hline Hydrogen chloride & 10 & $159 \sim 230$ & 270.32 & 3.28 & 0.021 & 1.97 & 0.43 & 3 \\
\hline Acetone & 27 & $275 \sim 471$ & 426.57 & 4.48 & 0.190 & 1.87 & 0.79 & 1 \\
\hline Grand average & & & & & & 1.31 & 0.68 & \\
\hline
\end{tabular}

$\mathrm{AAD} \%=\frac{100}{\mathrm{NP}} \Sigma_{\mathrm{i}}\left(\frac{\mathrm{X}^{\mathrm{cal}}-\mathrm{X}^{\exp }}{\mathrm{X}^{\exp }}\right) ; X=V^{\mathrm{v}}, V^{\mathrm{L}}$, or $P ; \mathrm{NP}=$ number of data points.

Experimental data sources: 1. Smith and Srivastava (1986); 2. Rossini et al. (1952); 3. Vargaftik (1975).

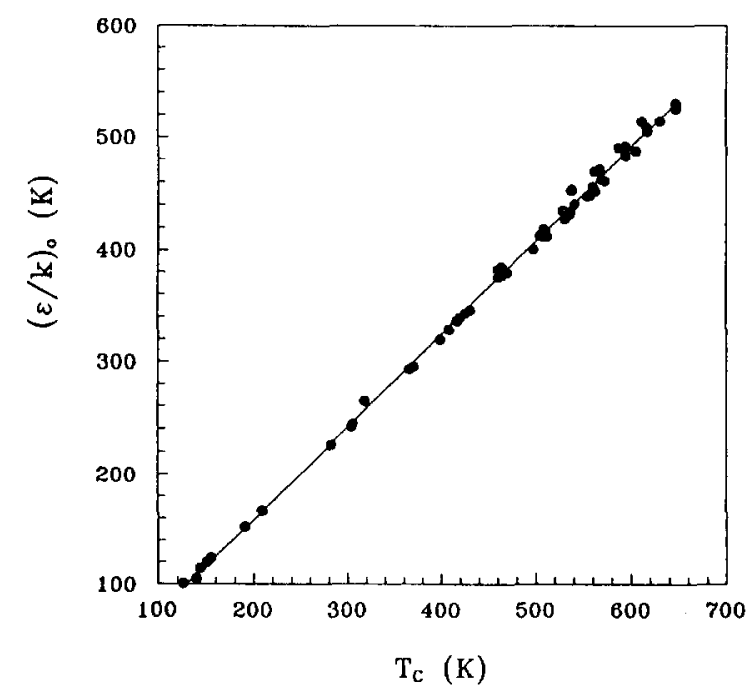

Fig. 1. Plot of $(\varepsilon / k)_{0}$ against the critical temperatures for nonpolar and weakly polar components including alkanes, cycloalkanes, alkenes, aromatics, alkanones, small molecules and halogenated alkanes. 


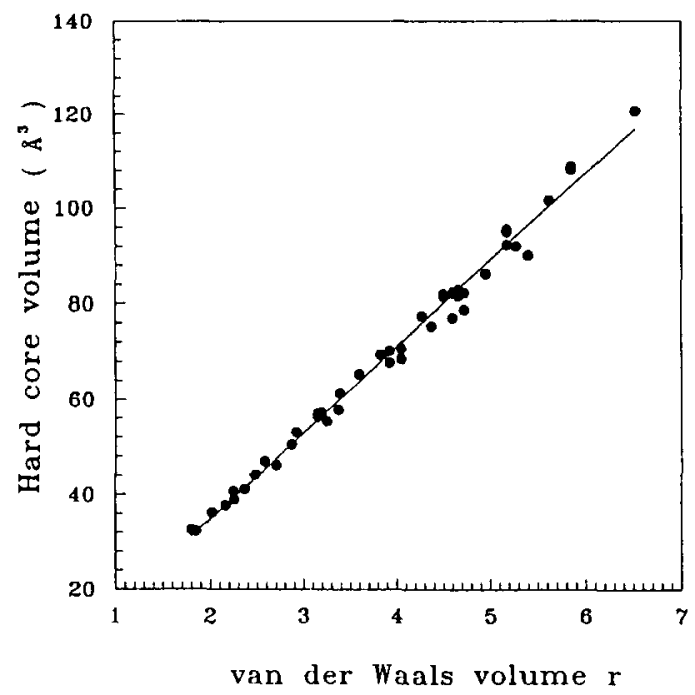

Fig. 2. Plot of the hard core volumes $\left(\pi \sigma^{3} / 6\right)$ against the Van der Waals volumes for nonpolar and weakly polar components including alkanes, cycloalkanes, alkenes, aromatics, alkanones and halogenated alkanes.

contribution method described by the UNIFAC model (Larsen et al., 1987). This indicated that $\sigma$ has a good physical significance as a molecular diameter parameter. The parameter $m$, which was used to correct the temperature-dependence of $(\epsilon / k)$, also increased linearly with the acentric factors for nonpolar and weakly polar pure fluids, as shown in Fig. 3. From the above observations, generalized

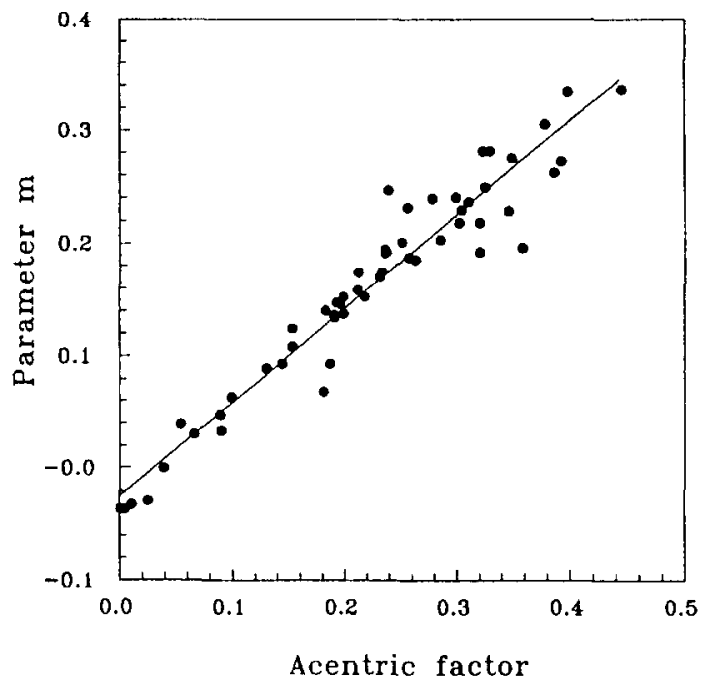

Fig. 3. Plot of parameter $m$ against the acentric factors for nonpolar and weakly polar components including alkanes, cycloalkanes, alkenes, aromatics, alkanones, small molecules and halogenated alkanes. 
correlations for pure component parameters of nonpolar and weakly polar fluids are proposed for the MPHS-EOS

$$
\begin{aligned}
& \left(\frac{\epsilon}{k}\right)_{0}=\frac{T_{\mathrm{c}}}{1.25-0.07 \omega} \\
& \sigma=\left(\frac{6 r}{0.055 \pi}\right)^{1 / 3}
\end{aligned}
$$

and

$$
m=0.9377 \omega-0.0477
$$

where $T_{c}, \omega$ and $r$ are the critical temperature, acentric factor and van der Waals volume, respectively. For nonpolar and weakly polar components, these generalized correlations gave satisfactory results in correlating the pure fluid properties. These correlation equations are useful in further predictive phase equilibrium calculations.

\section{Extension of the MPHS-EOS to fluid mixtures and VLE calculations}

The MPHS-EOS was extended to fluid mixtures in this work and the mixture EOS was written as

$$
\begin{aligned}
Z= & \frac{1+\zeta+\zeta^{2}-\zeta^{3}}{(1-\zeta)^{3}}-\frac{4.75}{3} \pi \sum_{i} \sum_{j} x_{i} x_{j} \rho_{i j}^{*} \\
& \times\left[\frac{1.45}{T_{i j}^{*}}-\exp \left(1-\phi_{i j}\right)\left(\frac{1.45}{T_{i j}^{*}}+1-\Omega_{i j}\right)\left(\frac{2 \sqrt{2}-\rho_{i j}^{*}-\sqrt{2} \phi_{i j}}{\sqrt{2}-\rho_{i j}^{*}}\right)\right]
\end{aligned}
$$
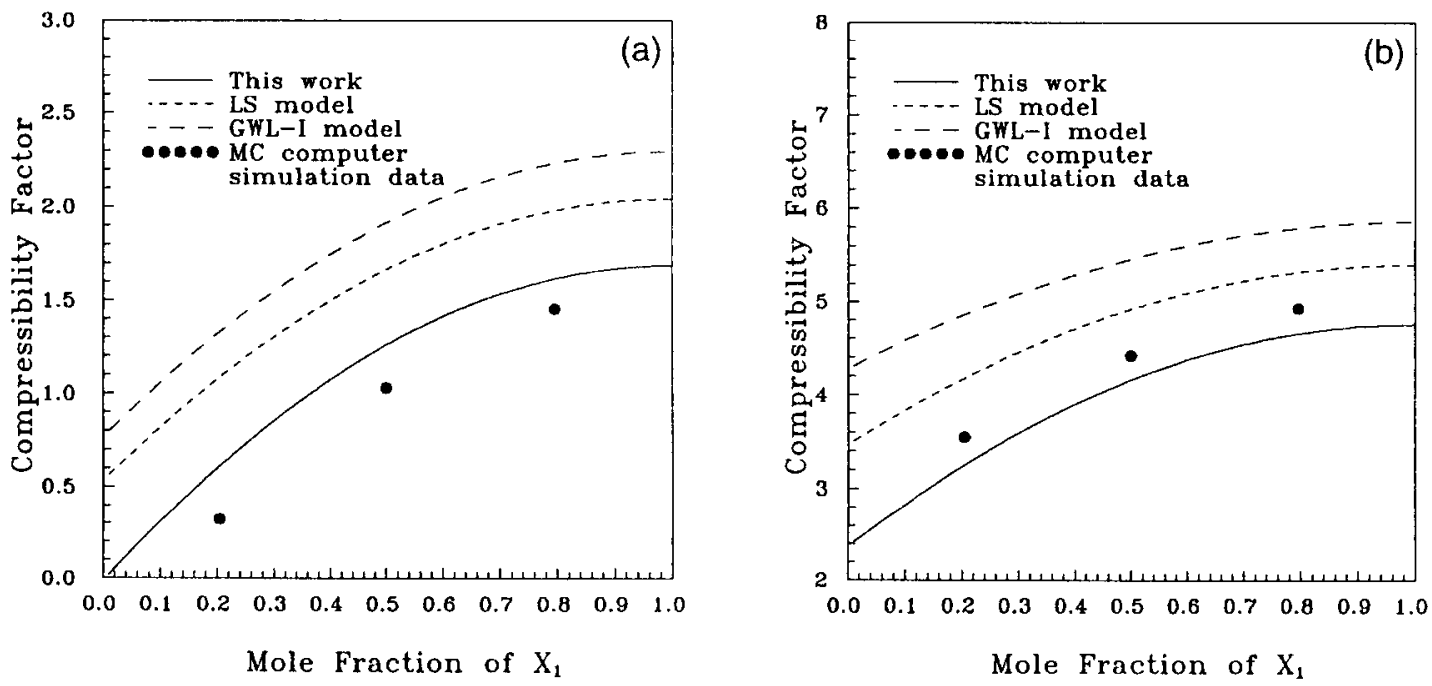

Fig. 4. Comparison of the calculated compressibility factors of SW fluid by various models (a) ( $\varepsilon_{11} / k T=0.5$, $\left.\varepsilon_{22} / k T=1.0, \varepsilon_{12} / k T=0.5, \rho \sigma^{3}=0.55, \sigma_{22} / \sigma_{11}=1\right)$ and (b) $\left(\varepsilon_{11} / k T=0.5, \varepsilon_{22} / k T=1.0, \varepsilon_{12} / k T=0.5, \rho \sigma^{3}=\right.$ $\left.0.80, \sigma_{22} / \sigma_{11}=1\right)$. MC computer simulation data source: Lee and Chao, 1987 . 

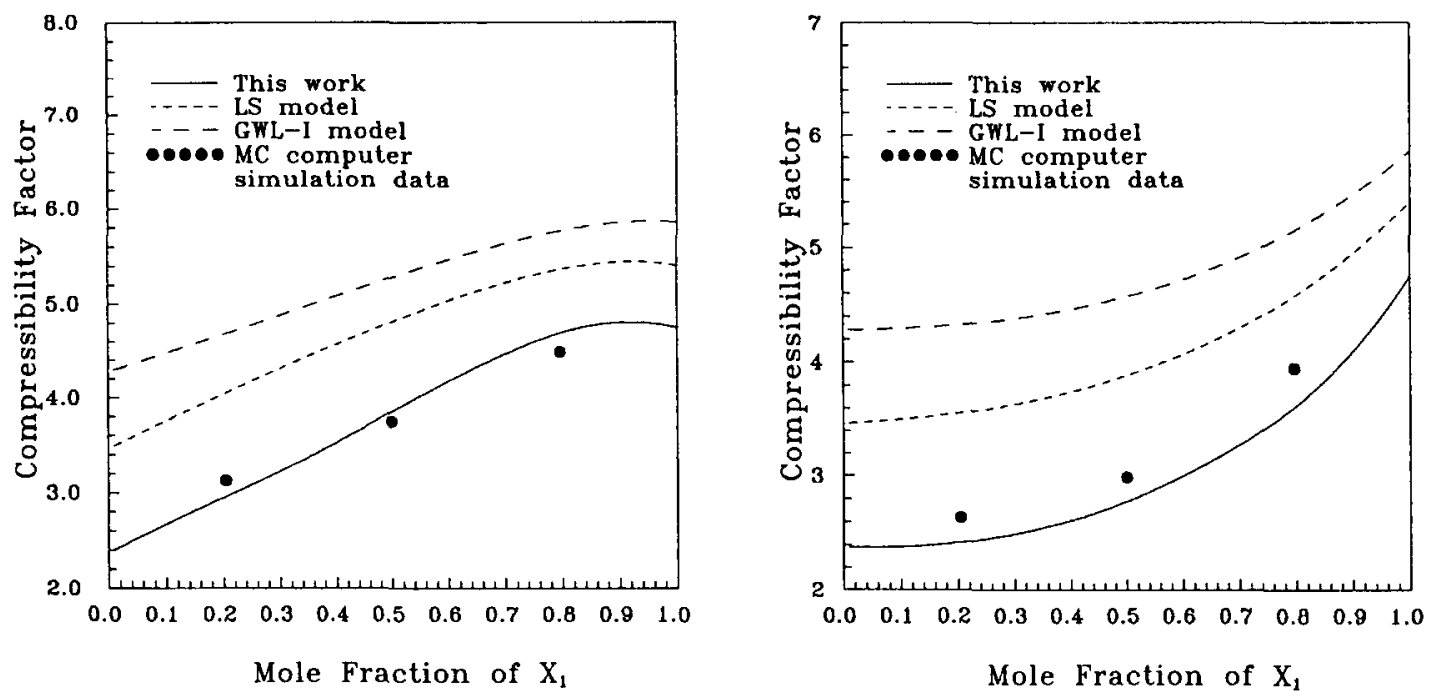

Fig. 5. Comparison of the calculated compressibility factors of SW fluid by various models (a) $\left(\varepsilon_{11} / k T=0.5\right.$, $\left.\varepsilon_{22} / k T=1.0, \varepsilon_{12} / k T=0.5, \rho \sigma^{3}=0.80, \quad \sigma_{22} / \sigma_{11}=1.50\right)$ and (b) $\left(\varepsilon_{11} / k T=0.5, \quad \varepsilon_{22} / k T=1.0, \varepsilon_{12} / k T=1.0\right.$, $\left.\rho \sigma^{3}=0.80, \sigma_{22} / \sigma_{11}=1.25\right)$. MC computer simulation data source: Lee and Chao, 1987 .

where $\rho_{i j}^{*}=\rho \sigma_{i j}^{3}, T_{i j}^{*}=(k T) /\left(\epsilon_{i j}\right), \Omega_{i j}=\exp \left(\left(\epsilon_{i j}\right) /(k T)\right), \zeta=(\pi \rho) /(6) \sum_{i} x_{i} \sigma_{i i}^{3}$ and $\phi_{i j}=(\sqrt{2}+$ $\left.\rho_{i j}^{*}\right) /\left(\sqrt{2}-\rho_{i j}^{*}\right)$. In contrast to the conventional cubic-type EOS, there was no need to introduce additional mixing rules in mixture property calculations. Using this EOS, we have calculated the compressibility factors for binary SW fluid mixtures and the results are presented in Figs. 4 and 5 . In these figures, the calculated compressibility factors were plotted against the compositions for various reduced densities and potential energy parameters. We also compared the results from other methods: those determined from the coordination number models of Lee and Sandler, 1987 (LS model) and of Guo et al., 1990a, Guo et al., 1990b (GWL-1 model). It is demonstrated that our calculated results have better agreement with the molecular simulation data than those from the other two EOS models. $\mathrm{Yu}$ and Chen (1995) have shown more comparisions of the calculated coordination numbers and compressibility factors of SW fluid mixtures from this MPHS-EOS with the molecular simulation data and those from other EOS models. The MPHS-EOS showed good ability in calculating the properties of the SW fluids. The extension of our calculations to real fluid mixtures on nonpolar and polar systems is presented in this study. In the real fluid mixture calculations, the cross-interaction properties were defined as

$$
\begin{aligned}
& \epsilon_{i j}=\sqrt{\epsilon_{i i} \epsilon_{j j}}\left(1-k_{i j}\right) \\
& \sigma_{i j}=\frac{\sigma_{i i}+\sigma_{j j}}{2}
\end{aligned}
$$

where $k_{i j}$ is a binary interaction parameter and was determined using experimental data. In this work, 
$k_{i j}$ was treated as a temperature-independent constant for a specific binary system. The expression of the fugacity coefficient from the MPHS-EOS is

$$
\begin{aligned}
\ln \hat{\Phi}_{i}= & 4.9742 \sum_{j} x_{j} \rho_{i j}^{*}\left[\exp \left(1-\phi_{i j}\right)\left(1-\Omega_{i j}+\frac{1.45}{T_{i j}^{*}}\right)-\frac{1.45}{T_{i j}^{*}}\right] \\
& +4.9742 \sum_{j} x_{j} \rho_{j i}^{*}\left[\exp \left(1-\phi_{j i}\right)\left(1-\Omega_{j i}+\frac{1.45}{T_{j i}^{*}}\right)-\frac{1.45}{T_{j i}^{*}}\right] \\
& +4.9742 \sum_{i} \sum_{j} x_{i} x_{j} \rho_{i j}^{*}\left[\exp \left(1-\phi_{i j}\right)\left(1-\Omega_{i j}+\frac{1.45}{T_{i j}^{*}}\right)\left(1-\phi_{i j}\right) \frac{\sqrt{2}}{\sqrt{2}-\rho_{i j}^{*}}\right] \\
& -\frac{\zeta(3 \zeta-4)}{(1-\zeta)^{2}}-\frac{2 \zeta-4}{(1-\zeta)^{3}}\left(\frac{\pi N_{A V}}{6 V} \sigma_{i i}^{3}\right)-\ln Z
\end{aligned}
$$

In the VLE calculations, the equal fugacity criterion was employed. We calculated the bubble point pressures and compositions of binary and ternary mixtures in this work, and compared our VLE calculation results using the MPHS-EOS with those from commonly used cubic-type Peng-Robinson (PR) EOS (Peng and Robinson, 1976) and the Patel-Teja (PT) EOS (Patel and Teja, 1982). For the cubic-type EOS, van der Waals mixing rules were used for mixture property calculations. One temperature-independent binary interaction parameter $l_{i j}$ was employed in evaluating the energy parameter of the cubic EOS

$$
a=\sum_{i} \sum_{j} x_{i} x_{j} \sqrt{a_{i} a_{j}}\left(1-l_{i j}\right)
$$

\section{VLE calculation results and discussion}

In this work, we calculated the VLE of 116 binary systems with similar to significantly different polarities. Three EOSs of PR, PT and MPHS were employed in this study and binary interaction parameters were empirically fitted for each EOS using experimental data. Table 2 presents typical calculation results for systems with similar polarities. It is observed that with the optimal binary interaction parameters, the MPHS-EOS yielded good results on bubble point pressures as well as on vapor phase compositions. The MPHS-EOS showed comparable results to those from the commonly used PR and PT cubic EOS. Table 3 shows typical VLE calculation results on binary mixtures with large differences in polarities. For the mixtures of components with large differences in polarities, it is demonstrated that the MPHS-EOS gave obviously better results. Taking the alcohol-paraffin binary mixtures for example, the PR- and PT-EOS methods have relatively larger absolute average deviations of more than $10 \%$ in bubble point pressure and vapor composition, even with their best-fitted binary parameters. The MPHS-EOS gave much smaller deviations in these systems which exhibited significant differences in polarity. The MPHS-EOS also yielded better results on other binary families as shown in Table 3 . The binary VLE calculation results are also shown graphically in this work. Fig. 6 shows the calculated Pxy curve for the binary mixture of ethanol and cyclohexane at 
Table 2

Comparison of the VLE calculation results for typical nonpolar and weakly polar binary mixtures by various equations of state with their optimal binary parameters

\begin{tabular}{|c|c|c|c|c|c|c|c|c|c|c|c|c|}
\hline \multirow[t]{2}{*}{ Component 1} & \multirow[t]{2}{*}{ Component 2} & \multirow{2}{*}{$\begin{array}{l}\text { No. of } \\
\text { data pts. }\end{array}$} & \multirow{2}{*}{$\begin{array}{l}\text { Temp. } \\
\text { range/K }\end{array}$} & \multicolumn{3}{|c|}{ Optimal $k_{i j}$ or $l_{i j}$} & \multicolumn{3}{|c|}{ AAD $\%$ in $P$} & \multicolumn{3}{|c|}{$\mathrm{AAD} \%$ in $y_{1}$} \\
\hline & & & & $\overline{\mathrm{PR}}$ & PT & MPHS & $\overline{\mathrm{PR}}$ & PT & $\overline{\text { MPHS }}$ & $\overline{\mathrm{PR}}$ & PTM & PHS \\
\hline 2-Methylpentane & $n$-Hexane & 9 & 298 & 0.001 & 0.004 & 0.004 & 0.39 & 0.34 & 0.31 & 1.55 & 0.81 & 0.64 \\
\hline 1-Heptene & $n$-Heptane & 14 & 328 & 0.015 & 0.018 & 0.005 & 2.35 & 2.45 & 0.40 & 3.23 & 3.57 & 0.40 \\
\hline Benzene & Cyclohexane & 38 & $298 \sim 343$ & 0.022 & 0.023 & 0.020 & 1.58 & 1.33 & 0.35 & 1.30 & 1.12 & 1.06 \\
\hline Chloroform & Benzene & 24 & $308 \sim 323$ & -0.015 & -0.014 & -0.014 & 1.25 & 1.25 & 0.96 & 2.24 & 2.07 & 1.47 \\
\hline Acetone & Toluene & 15 & 318 & 0.035 & 0.036 & 0.019 & 1.98 & 1.50 & 0.71 & 1.06 & 0.93 & 0.54 \\
\hline$n$-Heptane & Pyridine & 13 & 353 & 0.035 & 0.038 & 0.050 & 1.08 & 1.43 & 0.68 & 3.84 & 4.05 & 1.01 \\
\hline$n$-Pentane & Acetone & 32 & $238 \sim 298$ & 0.091 & 0.091 & 0.082 & 4.38 & 4.78 & 2.69 & 2.78 & 2.74 & 2.33 \\
\hline Acetone & $n$-Hexane & 56 & $253 \sim 328$ & 0.087 & 0.089 & 0.079 & 3.81 & 3.69 & 2.50 & 5.30 & 5.27 & 2.04 \\
\hline Acetone & $n$-Heptane & 7 & 323 & 0.099 & 0.100 & 0.073 & 5.38 & 5.19 & 1.51 & 3.68 & 3.52 & 1.03 \\
\hline Diethyl ether & Acetone & 11 & 303 & 0.034 & 0.033 & 0.026 & 1.57 & 2.0 & 0.86 & 2.94 & 2.80 & 2.01 \\
\hline Ethanol & 1-Propanol & 36 & $323 \sim 353$ & 0.003 & 0.014 & 0.005 & 2.31 & 1.02 & 1.13 & 4.53 & 0.82 & 0.89 \\
\hline Ethanol & 1-Pentanol & 10 & $353 \sim 406$ & 0.005 & 0.012 & 0.002 & 3.71 & 4.32 & 2.56 & 5.01 & 4.62 & 3.57 \\
\hline Grand average & & & & & & & 2.64 & 2.48 & 1.42 & 3.34 & 2.77 & 1.49 \\
\hline
\end{tabular}

Data sources: Gmehling et al., 1978 and subsequent equilibrium data collections. 
Table 3

Comparison of the VLE calculation results for typical polar mixtures by various equations of state with their optimal binary parameters

\begin{tabular}{|c|c|c|c|c|c|c|c|c|c|c|c|c|}
\hline \multirow[t]{2}{*}{ Component 1} & \multirow[t]{2}{*}{ Component 2} & \multirow{2}{*}{$\begin{array}{l}\text { No. of } \\
\text { data pts. }\end{array}$} & \multirow{2}{*}{$\begin{array}{l}\text { Temp. } \\
\text { range/K }\end{array}$} & \multicolumn{3}{|c|}{ Optimal $k_{i j}$ or $l_{i j}$} & \multicolumn{3}{|c|}{ AAD\% in $P$} & \multicolumn{3}{|c|}{$\mathrm{AAD} \%$ in $y_{1}$} \\
\hline & & & & $\overline{\mathrm{PR}}$ & $\mathrm{PT}$ & MPHS & $\overrightarrow{\mathrm{PR}}$ & PT & MPHS & $\overline{P R}$ & $\mathrm{PT}$ & MPHS \\
\hline $\overrightarrow{\text { 1-Pentene }}$ & Methanol & 13 & $300 \sim 322$ & 0.016 & 0.017 & 0.072 & 15.12 & 15.38 & 6.16 & 6.50 & 6.82 & 3.77 \\
\hline$n$-Pentane & Methanol & 22 & $303 \sim 335$ & 0.017 & 0.019 & 0.095 & 9.83 & 10.16 & 9.07 & 12.34 & 12.36 & 10.03 \\
\hline Methanol & Cyclohexane & 25 & $318 \sim 328$ & 0.079 & 0.082 & 0.105 & 12.27 & 12.66 & 5.31 & 15.29 & 15.54 & 6.23 \\
\hline$n$-Hexane & Methanol & 12 & 318 & 0.023 & 0.026 & 0.095 & 10.54 & 10.82 & 9.19 & 14.46 & 14.71 & 10.58 \\
\hline Methanol & $n$-Heptane & 7 & $332 \sim 334$ & 0.025 & 0.033 & 0.089 & 14.16 & 14.23 & 4.03 & 10.67 & 10.57 & 5.68 \\
\hline Ethanol & Isooctane & 35 & $273 \sim 323$ & 0.003 & 0.006 & 0.080 & 15.39 & 16.25 & 7.82 & 23.85 & 23.73 & 14.15 \\
\hline 1-Propanol & $n$-Decane & 12 & 368 & 0.053 & 0.058 & 0.059 & 13.71 & 11.22 & 5.83 & 4.65 & 4.25 & 1.58 \\
\hline Methanol & Toluene & 14 & $337 \sim 363$ & 0.111 & 0.116 & 0.085 & 15.51 & 15.42 & 2.76 & 9.54 & 9.79 & 4.07 \\
\hline Ethanol & $p$-Xylene & 15 & $352 \sim 389$ & 0.087 & 0.092 & 0.079 & 11.62 & 10.19 & 3.02 & 7.22 & 7.21 & 5.16 \\
\hline Methanol & $\mathrm{CCl}_{4}$ & 18 & $308 \sim 328$ & 0.082 & 0.085 & 0.083 & 10.82 & 11.20 & 5.36 & 25.45 & 25.68 & 12.86 \\
\hline Methanol & Ethyl acetate & 53 & $313 \sim 333$ & 0.010 & 0.015 & 0.030 & 4.13 & 4.30 & 1.77 & 7.90 & 7.72 & 3.43 \\
\hline Ethyl formate & Ethanol & 12 & 318 & 0.055 & 0.055 & 0.047 & 2.46 & 2.85 & 0.76 & 2.68 & 2.53 & 1.05 \\
\hline Ethanol & 2-Butanone & 14 & 328 & 0.025 & 0.028 & 0.033 & 1.70 & 1.90 & 0.84 & 2.60 & 2.54 & 2.31 \\
\hline Diethyl ether & Ethanol & 19 & 298 & -0.013 & -0.013 & 0.034 & 6.58 & 6.42 & 1.94 & 2.73 & 3.11 & 0.81 \\
\hline Grand average & & & & & & & 10.69 & 9.31 & 4.23 & 11.50 & 11.52 & 6.33 \\
\hline
\end{tabular}

Data sources: Gmehling et al. (1978) and subsequent equilibrium data collections. 


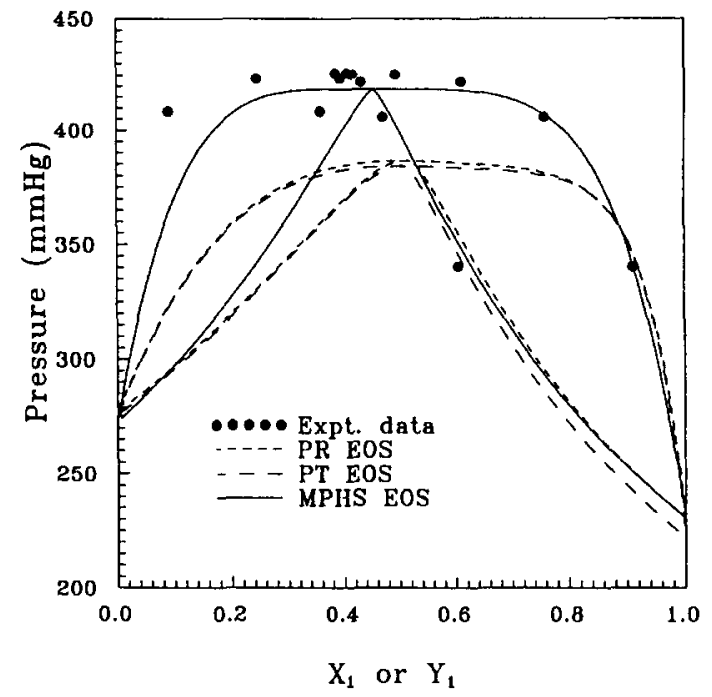

Fig. 6. Comparison of VLE calculation results by various EOS for the binary mixture of ethanol (1) and cyclohexane (2) at $323 \mathrm{~K}$.

$323 \mathrm{~K}$. This mixture is strongly nonideal with an azeotrope. It is shown that the PR- and PT-EOS gave appreciable calculated errors even with their optimally fitted binary parameters. The MPHS-EOS yielded reasonably good agreement with the experimental data. Similar results are shown in Fig. 7 where the MPHS-EOS gave better VLE calculation results than those from the cubic EOS for the binary mixture of methanol and benzene at $318 \mathrm{~K}$. Table 4 presents a comparison of the optimally fitted binary parameters of the three EOSs in our VLE calculations. For each family of binary

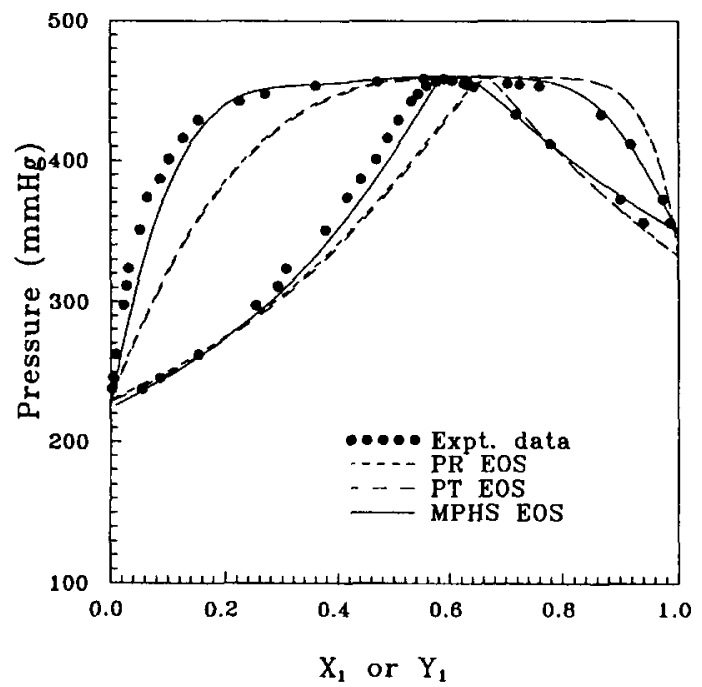

Fig. 7. Comparison of VLE calculation reults by various EOS for the binary mixture of methanol (1) and benzene ( 2 ) at 318 K. 
Table 4

Comparison of the scattering of binary parameters of various EOS methods

\begin{tabular}{|c|c|c|c|c|c|}
\hline \multirow{2}{*}{$\begin{array}{l}\text { Family of binary } \\
\text { mixtures }\end{array}$} & \multirow{2}{*}{$\begin{array}{l}\text { No. of } \\
\text { data pts. }\end{array}$} & \multirow{2}{*}{$\begin{array}{l}\text { Temp. } \\
\text { range } / \mathrm{K}\end{array}$} & \multicolumn{3}{|c|}{$100\left(\operatorname{Max} k_{i j}-\operatorname{Min} k_{i j}\right) / \operatorname{Max} k_{i j}$} \\
\hline & & & $\overline{\mathrm{PR}}$ & PT & MPHS \\
\hline Alkane + benzene & 136 & $298 \sim 353$ & 119 & 122 & 15 \\
\hline Alkane + toluene & 67 & $298 \sim 398$ & 125 & 113 & 75 \\
\hline Alkane + pyridine & 64 & $353 \sim 370$ & 69 & 71 & 12 \\
\hline Alkane + acetone & 155 & $238 \sim 328$ & 21 & 20 & 11 \\
\hline Alkane + methanol & 85 & $297 \sim 335$ & 83 & 82 & 32 \\
\hline Alkane + ethanol & 201 & $263 \sim 433$ & 98 & 96 & 22 \\
\hline Alkane + 1-propanol & 80 & $318 \sim 368$ & 50 & 57 & 29 \\
\hline Aromatic + methanol & 66 & $308 \sim 363$ & 25 & 27 & 5 \\
\hline Aromatic + ethanol & 69 & $308 \sim 389$ & 17 & 13 & 13 \\
\hline Aromatic +1 -propanol & 98 & $303 \sim 377$ & 50 & 45 & 27 \\
\hline Alkanone + methanol & 81 & $308 \sim 351$ & 157 & 109 & 52 \\
\hline Alkanone + ethanol & 67 & $305 \sim 361$ & 27 & 28 & 14 \\
\hline Ester + methanol & 132 & $293 \sim 333$ & 81 & 70 & 36 \\
\hline Ester + ethanol & 115 & $298 \sim 353$ & 70 & 70 & 57 \\
\hline Ester + 1-propanol & 61 & $313 \sim 371$ & 72 & 60 & 51 \\
\hline
\end{tabular}

$\operatorname{Max} k_{i j}$ or min $k_{i j}$ : maximum or minimum binary parameters used on VLE calculations for each EOS.

mixtures, the scattering of the binary parameters were evaluated and are shown in Table 4. It is observed that the scattering of the binary parameters of the MPHS-EOS is less than that of other EOSs. This advantage makes it promising either to extend the MPHS-EOS on predictive VLE calculations where estimated binary parameters of a specific family of mixture may be used, or to apply the MPHS-EOS to multicomponent VLE computations.

Table 5

Typical ternary systems studied in this research

\begin{tabular}{|c|c|c|c|c|c|c|}
\hline No. & Component 1 & Component 2 & Component 3 & $\begin{array}{l}\text { No. of } \\
\text { data pts. }\end{array}$ & $\begin{array}{l}\text { Temperature } \\
\text { range } / \mathrm{K}\end{array}$ & $\begin{array}{l}\text { Pressure } \\
\text { range } / \mathrm{mm} \mathrm{Hg}\end{array}$ \\
\hline 1 & Acetone & $\mathrm{CHCl}_{3}$ & Methanol & 36 & $329 \sim 335$ & 760 \\
\hline 2 & $\mathrm{CHCl}_{3}$ & Methanol & Benzene & 35 & $329 \sim 335$ & 760 \\
\hline 3 & Methanol & Ethanol & Methyl acetate & 6 & $331 \sim 338$ & 760 \\
\hline 4 & Acetone & Methanol & Cyclohexane & 63 & $308 \sim 328$ & $358 \sim 868$ \\
\hline 5 & Methanol & Benzene & Cyclohexane & 19 & $312 \sim 328$ & $334 \sim 763$ \\
\hline 6 & $\mathrm{CCl}_{4}$ & Ethanol & Benzene & 36 & 323 & $355 \sim 426$ \\
\hline 7 & Ethanol & $\mathrm{CHCl}_{3}$ & $n$-Hexane & 108 & $308 \sim 328$ & $240 \sim 701$ \\
\hline 8 & Ethanol & $\mathrm{CHCl}_{3}$ & $n$-Hexane & 36 & $331 \sim 337$ & 760 \\
\hline 9 & Acetone & Ethanol & $n$-Hexane & 21 & 328 & $636 \sim 884$ \\
\hline 10 & Acetone & Ethanol & $n$-Hexane & 21 & $324 \sim 334$ & 760 \\
\hline 11 & Benzene & Ethanol & $n$-Hexane & 7 & $333 \sim 339$ & 760 \\
\hline 12 & Ethanol & Benzene & $n$-Heptane & 148 & $306 \sim 352$ & $180 \sim 760$ \\
\hline 13 & Toluene & Cyclohexane & Ethanol & 19 & 323 & $249 \sim 408$ \\
\hline 14 & Benzene & $n$-Heptane & 1-Propanol & 77 & 348 & $450 \sim 713$ \\
\hline
\end{tabular}

Data sources: Gmehling et al. (1978) and subsequent equilibrium data collections. 
Table 6

Comparison of the VLE calculation results for ternary systems from various equation of state

\begin{tabular}{|c|c|c|c|c|c|c|c|c|c|}
\hline \multirow[t]{2}{*}{ No. } & \multicolumn{3}{|c|}{ AAD\% in $P$} & \multicolumn{3}{|c|}{$\triangle \mathrm{AD} \%$ in $y_{1}$} & \multicolumn{3}{|c|}{$\mathrm{AAD} \%$ in $y_{2}$} \\
\hline & PR & $\mathrm{PT}$ & MPHS & PR & PT & MPHS & PR & PT & MPHS \\
\hline 1 & 2.62 & 2.64 & 4.61 & 11.64 & 11.62 & 14.01 & 10.98 & 10.58 & 8.95 \\
\hline 2 & 7.57 & 7.91 & 3.18 & 12.13 & 11.89 & 9.74 & 14.82 & 14.82 & 9.53 \\
\hline 3 & 2.84 & 2.61 & 7.83 & 8.54 & 8.90 & 7.36 & 8.22 & 7.12 & 5.14 \\
\hline 4 & 5.13 & 5.44 & 0.94 & 7.46 & 7.41 & 4.59 & 13.16 & 13.46 & 6.33 \\
\hline 5 & 17.61 & 17.91 & 3.51 & 18.67 & 18.85 & 5.31 & 19.92 & 20.18 & 6.52 \\
\hline 6 & 7.63 & 8.92 & 3.77 & 8.77 & 8.69 & 4.47 & 15.96 & 15.66 & 8.46 \\
\hline 7 & 13.62 & 14.87 & 6.04 & 25.65 & 25.04 & 14.73 & 11.82 & 13.05 & 6.13 \\
\hline 8 & 14.18 & 15.34 & 5.16 & 26.51 & 26.30 & 16.07 & 12.22 & 13.42 & 6.56 \\
\hline 9 & 11.09 & 12.05 & 1.58 & 17.82 & 19.11 & 4.97 & 15.34 & 15.33 & 5.00 \\
\hline 10 & 10.83 & 11.81 & 2.01 & 17.92 & 19.23 & 5.46 & 17.70 & 17.77 & 8.23 \\
\hline 11 & 17.45 & 18.30 & 9.05 & 20.08 & 21.32 & 7.17 & 37.87 & 38.67 & 21.07 \\
\hline 12 & 9.54 & 9.29 & 4.97 & 22.15 & 21.80 & 12.72 & 18.38 & 17.90 & 10.89 \\
\hline 13 & 12.05 & 12.25 & 4.33 & 12.04 & 10.89 & 4.93 & 14.66 & 14.24 & 6.22 \\
\hline 14 & 7.95 & 10.10 & 5.86 & 9.24 & 9.18 & 5.57 & 10.95 & 10.40 & 7.23 \\
\hline Gran & g. 9.69 & 10.39 & 4.48 & 17.20 & 17.04 & 9.94 & 14.66 & 14.74 & 8.11 \\
\hline
\end{tabular}

Table 5 presents typical ternary systems where VLE calculations were made in this study. In the ternary calculations, binary parameters determined from binary VLE computations were used directly. Table 6 shows typical ternary calculated results and the comparisons among three EOS methods. It is demonstrated that the MPHS-EOS gave better results in bubble point pressures and vapor phase compositions for most of the ternary systems. It is again shown that the MPHS-EOS yielded significant improvement on the mixtures where constituents have large differences in polarities.

\section{Conclusion}

A new MPHS-EOS was used in this study on VLE calculations of binary and ternary fluid mixtures. EOS parameters of pure fluids were evaluated and generalized correlations for these parameters were obtained for nonpolar and weakly polar fluids. The MPHS-EOS presented satisfactory VLE calculation results on binary and ternary systems. The MPHS-EOS showed significant improvement on the VLE calculations for fluid mixtures having large differences in polarities. The MPHS-EOS showed better VLE calculation results than those from the commonly used PR and PT cubic EOS. The MPHS-EOS also had less scattering of the optimally fitted binary parameters for a specific family of mixtures than the other cubic EOSs.

\section{Nomenclature}

a cubic-type EOS energy parameters

$f \quad$ fugacity 
$k \quad$ Boltzmann's constant

$k_{i j}, l_{i j}$ binary interaction parameter

$m$ temperature-dependent parameter in Eq. (6)

$N \quad$ number of molecules

$N_{\mathrm{AV}} \quad$ Avogadro's number

$P \quad$ pressure

$Q \quad$ object function

$r \quad$ van der Waals volume parameter

$T$ absolute temperature

$T_{\mathrm{c}} \quad$ critical temperature

$T^{*} \quad$ dimensionless parameter of $k T / \epsilon$

$V \quad$ volume

$x, y$ mole fraction

$Z \quad$ compressibility factor

\subsection{Greek letters}

$\epsilon \quad$ depth parameter of the SW potential

$\Phi$ fugacity coefficient

$\phi \quad$ density function defined in Eq. (2)

$\lambda \quad$ width parameter of the SW potential

$\rho \quad$ number density

$\rho^{*} \quad$ reduced density, $\rho \sigma^{3}$

$\sigma \quad$ molecular diameter

$\Omega \quad$ dimensionless parameter in Eq. (11)

$\zeta$ dimensionless parameter of $(\pi \rho / 6) \Sigma x_{i} \sigma_{i}{ }^{3}$

$\omega \quad$ acentric factor

\subsection{Subscripts}

c critical properties

$i, j$ component $i$ or $j$

1,2 component 1 or 2

\subsection{Superscripts}

cal calculated

exp experimental

L liquid phase

$\mathrm{V}$ vapor phase

VP vapor pressure

* dimensionless properties 


\section{Acknowledgements}

The authors are grateful to the National Science Council of the Republic of China for supporting this research.

\section{References}

Carnahan, N.F. and Starling, K.E., 1969. Equation of state for non-attracting spheres. J. Chem. Phys., 51: 635-636.

Gmehling, J., Onken, U., Artt, W., Grenzheuser, P., Weidlich, U., Kolbe, B. and Rarey-Nies, J., 1978-1988. Vapor-Liquid Equilibrium Data Collection. DECHEMA, Frankfurt, Germany.

Guo, M.-X., Wang, W.-C. and Lu, H.-Z., 1990(a). Equations of state for pure and mixture square-well fluids. I. Coordination number models. Fluid Phase Equilibria, 60: 37-45.

Guo, M.-X., Wang, W.-C. and Lu, H.-Z., 1990(b). Equations of state for pure and mixture square-well fluids. II. Equations of state. Fluid Phase Equilibria, 60: 221-237.

Larsen, B.L., Rasmussen, P. and Fredenslund, A., 1987. A modified UNIFAC group-contribution model for prediction of phase equilibria and heats of mixing. Ind. Eng. Chem. Res., 26: 2274-2286.

Lee, K.-H., Lombardo, M. and Sandler, S.I., 1985. The generalized van der Waals partition function. II. Application to the square-well fluid. Fluid Phase Equilibria, 21: 177-196.

Lee, K.-H., Sandler, S.I. and Patel, N.C., 1986. The generalized van der Waals partition function. III. Local composition models for a mixture of equal size square-well fluid. Fluid Phase Equilibria, 25: 31-49.

Lee, K.-H. and Sandler, S.I., 1987. The generalized van der Waals partition function. IV. Local composition models for mixtures of unequal-size molecules. Fluid Phase Equilibria, 34: 113-147.

Lee, K.-H., Dodd L.R. and Sandler, S.I., 1989. The generalized van der Waals partition function. V. Mixtures of square-well fluids of different sizes and energies. Fluid Phase Equilibria, 50: 53-77.

Lee, R.J. and Chao, K.C., 1987. Coordination number and thermodynamics of square-well fluid mixtures. Molecular Phys. 61: 1431-1442.

Patel, N.C. and Teja, A.S., 1982. A new cubic equation of state for fluid and fluids mixtures. Chem. Eng. Sci., 37:463-473. Peng, D.-Y. and Robinson, D.B., 1976. A new two-constant equation of state. Ind. Eng. Chem., Fundam., 15: 59-64.

Rossini, F.D., Pitzer, K.S., Arnett, R.L., Braun, R.M. and Pimental, G.C., 1952. Selected values of properties of hydrocarbons and related compounds. API Project 44, Canegie, Pittsburgh.

Sandler, S.I., 1985. The generalized van der Waals partition function. I. Basic theory. Fluid Phase Equilibria, 19: 233-257.

Smith, B.D. and Srivastava, R., 1986. Thermodynamic Data for Pure Compounds. Elsevier, Amsterdam.

Vargaftik, N.B., 1975. Tables on the Thermodynamic Properties of Liquids and Gases. 2nd. edn., Hemisphere Publishing Corp. Washington, D.C.

Yu, M.-L. and Chen, Y.-P., 1995. An equation of state from a new coordination number model. Fluid Phase Equilibria, 111: $37-51$. 